\title{
Quantum Stoner-Wohlfarth Model
}

\author{
Takuya Hatomura, ${ }^{1, *}$ Bernard Barbara, ${ }^{2,3}$ and Seiji Miyashita, ${ }^{1,4}$ \\ ${ }^{1}$ Department of Physics, Graduate School of Science, The University of Tokyo, 7-3-1 Hongo, Bunkyo-Ku, Tokyo 113-0033, Japan \\ ${ }^{2}$ Institut Néel, CNRS, F-38042 Grenoble, France \\ ${ }^{3}$ Université Grenoble-Alpes/Institut Néel, F-38042 Grenoble, France \\ ${ }^{4}$ CREST, JST, 4-1-8 Honcho Kawaguchi, Saitama 332-0012, Japan
}

(Received 18 March 2015; published 22 January 2016)

\begin{abstract}
The quantum mechanical counterpart of the famous Stoner-Wohlfarth model - an easy-axis magnet in a tilted magnetic field - is studied theoretically and through simulations as a function of the spin size $S$ in a sweeping longitudinal field. Beyond the classical Stoner-Wohlfarth transition, the sweeping field-induced adiabatic change of states slows down as $S$ increases, leading to a dynamical quantum phase transition. This result gives us new insights to describe the collapse of the metastability from the viewpoint of a critical phenomenon associated with the Landau-Zener tunneling gaps. Furthermore, a beating of the amplitude of the magnetization (the spin-length fidelity) is discovered after the Stoner-Wohlfarth transition. The period of the beating, confirmed analytically, arises from a new type of quantum phase factor.
\end{abstract}

DOI: 10.1103/PhysRevLett.116.037203

The Stoner-Wohlfarth (SW) model [1] is a classical model giving the field values at which a spin with uniaxial anisotropy switches under a tilted magnetic field applied in the opposite hemisphere. This is a discontinuous metastable to stable transition, in the limit of infinite spin. The quantum counterpart of this model has been studied from the viewpoint of quantum tunneling by making use of the WKB approach in zero longitudinal field $[2,3]$. The wellknown phenomena of the stepwise magnetization curves observed in the single molecule magnet $\mathrm{Mn}_{12}$-ac demonstrated quantum tunneling at avoided crossings of opposite magnetization levels [4,5]. Observed discreteness was a result of the finite value of the molecule spin $(S=10)$. The phenomena have been studied from the viewpoint of quantum tunneling, including theoretical approaches based on the Landau-Zener (LZ) transition [6,7], which was also observed in experiments [8]. Some quantum aspects of the spinodal transition have been studied in the transverse Ising chain with a sweeping longitudinal field [9] in which a relatively fast sweeping case was studied.

The present study gives a more direct quantum effect on the collapse of the metastable state. We study the quantum SW model numerically in the presence of a fixed transverse field and a sweeping longitudinal field in the limit of large spins (going from $S=20$ to 320). When $S$ increases, we find, from a microscopic viewpoint, how the densification of the energy level structure, leading to a suppression of discreteness, affects the collapse of metastability and modifies the spin dynamics. We study more particularly the quantum critical properties appearing near the end of metastability of the classical model. Furthermore, a precession beating is discovered which is confirmed analytically and interpreted in terms of a new type of quantum phase factor.
In order to catch the properties of our model in the $S \rightarrow \infty$ limit properly, we introduce the normalized quantum spin operators with a modified commutation relation:

$$
s_{\alpha}=\frac{S_{\alpha}}{S}, \quad(\alpha=x, y, z), \quad\left[s_{\alpha}, s_{\beta}\right]=\frac{i}{S} \epsilon_{\alpha \beta \gamma} s_{\gamma} .
$$

The corresponding SW Hamiltonian, with uniaxial anisotropy, transverse field (fixed), and longitudinal field (sweeping at the time rate $c$ ), is written as [10]

$$
\mathcal{H}=-D s_{z}^{2}-H_{x} s_{x}-H_{z} s_{z}, \quad H_{z}=H_{z}^{(0)}-c t .
$$

In Eqs. (1) and (2) and hereafter, we set $g \mu_{B}=1$ and $\hbar=1$.

The time evolution of the normalized quantum spin operators is given by

$$
\begin{aligned}
\frac{d s_{x}}{d t} & =\frac{1}{S}\left\{D\left(s_{y} s_{z}+s_{z} s_{y}\right)+H_{z} s_{y}\right\}, \\
\frac{d s_{y}}{d t} & =\frac{1}{S}\left\{-D\left(s_{z} s_{x}+s_{x} s_{z}\right)-H_{z} s_{x}+H_{x} s_{z}\right\}, \\
\frac{d s_{z}}{d t} & =-\frac{1}{S} H_{x} s_{y} .
\end{aligned}
$$

The corresponding time evolution of the usual (classical) SW model comes from the torque equation for a unit vector of magnetization $\boldsymbol{m}, d \boldsymbol{m} / d t=-\boldsymbol{m} \times \boldsymbol{H}_{\text {eff }}$ with the effective field $\boldsymbol{H}_{\text {eff }}=-\partial E_{\mathrm{SW}} / \partial \boldsymbol{m}=\left(H_{x}, 0,2 D m_{z}+H_{z}\right)$. Comparing this with Eq. (3), we find that the dynamics becomes the same if we ignore the commutation relations among spin operators in Eq. (3). But the time in the quantum system should be rescaled as

$$
\tau \equiv t / S
$$


to study correspondence with the classical dynamics. Then, the sweeping rate $c$ must be rescaled as $c=v / S$, where $v$ is the sweeping rate of the corresponding classical model.

The motion of a spin of the classical SW model $\boldsymbol{m}(t)$ draws a trajectory on the unit sphere. If $H_{z}$ and $H_{x}$ are weak compared to the anisotropy $D$, the system has a metastable state in which $m_{z}$ is antiparallel to the direction of $H_{z}$. If $H_{z}$ and $H_{x}$ increase and the $\mathrm{SW}$ condition $(2 D)^{2 / 3}=$ $\left(H_{x}\right)^{2 / 3}+\left(H_{z}\right)^{2 / 3}$ is satisfied, the metastable state becomes unstable.

Figure 1 shows the classical motion of magnetization $\left(m_{x}(t), m_{y}(t), m_{z}(t)\right)$ under a sweeping longitudinal field. It starts from the metastable point at $H_{z}(0)=4$ (arrow 1 at $t=0)$. The motion in this process is adiabatic up to the $\mathrm{SW}$ point (arrow 2 at $t=t_{\mathrm{SW}}$ ). At $t=t_{\mathrm{SW}}$ the irreversible magnetization jump takes place. It is followed by a precessionlike motion around the effective field $\boldsymbol{H}_{\text {eff }}(t)$.

To characterize the quantum SW transition of a large spin $S$, we first investigate the energy-level structure as a function of $H_{z}$. The example for $S=20$ is given in Fig. 2, in which $2 S+1$ levels with positive and negative magnetization slopes intercept at avoided-level crossings with gaps. Under the sweeping field $H_{z}=1-c t$ (applied at $H_{z}=1, t=0$ ), the ground state remains fully occupied until $H_{z}=0$, where the first avoided-level crossing (between $S$ and $-S$ ) is reached. If the reciprocal sweeping time was much smaller than the gap at this point, the motion would be adiabatic and the spin reversal $(S \rightarrow-S)$ would take place. However, in practice, this change hardly occurs because, with realistic parameters, the gap is vanishingly small for a large $S$, and thus only a very small fraction of the population scatters from $+S$ to $-S$. The rest remains on the line continuing the $H_{z}>0$ ground state into the $H_{z}<0$ region. This $H_{z}<0$ line corresponds to the classical metastable state (trajectory between points 1 and 2 in Fig. 1) and is called here the metastable branch. This branch crosses the levels of slopes $M=-S,-S+1$, $-S+2, \ldots,-S+k, \ldots$, successively (see Fig. 2). At each crossing, some population of the metastable branch scatters to the state of negative magnetization. For a finite value of $S$, say $S=10$ for $\mathrm{Mn}_{12}$ and $\mathrm{Fe}_{8}$, the so-called resonant

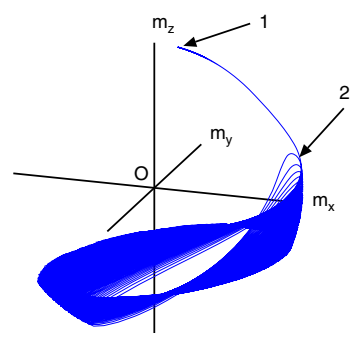

FIG. 1. A classical trajectory under the sweeping field $H_{z}=$ $4-c t(c=0.01)$ for $D=1, H_{x}=1$. Arrow 1 indicates the starting point which is a metastable fixed point for $H_{z}=4$, and arrow 2 the point of the SW transition followed by precession around the magnetic field. tunneling takes place at each crossing depending on the energy gap at the crossing. For the one-way sweeping of the field from the ground state, the scatterings at the successive anticrossings can be regarded as the sum of independent LZ events [11]. In addition, the events are independent because the gap of a given anticrossing $\Delta E$ is much smaller than the separation of two anticrossings $D / S$. The reduced LZ probability of remaining in the metastable state at the $k$ th avoided level crossing is given by

$$
p_{k}^{(S)}=\exp \left(-\frac{\pi\left(\Delta E_{k}^{(S)}\right)^{2}}{2 \hbar c \Delta m_{k}^{(S)}}\right)
$$

where $\Delta E_{k}^{(S)}$ is the energy gap at the field $H_{z}^{k}$ resulting from the mixing of the spins $S$ (metastable branch) with $-S+(k-1) \quad$ (intercepted branches), and $\Delta m_{k}^{(S)} \simeq$ $(2 S-k) / S$ is the reduced difference of magnetization slopes of the two states. As $S$ becomes larger, the spectrum associated with a normalized spin densifies because the number of the eigenvalues $(2 S+1)$ increases. For example, if we increase $S$ from $S$ to $2 S$, a new eigenvalue appears between two consecutive eigenvalues of the initial spectrum of $S$. In the continuous limit, the amount of scattering within a given field interval must be nearly the same, leading to the condition $p_{2 k}^{(2 S)} p_{2 k+1}^{(2 S)} \simeq p_{k}^{(S)}$ for successive LZ transitions, i.e., to the expression of the scaled gap $\Delta E_{\text {scaled }}=S \Delta E_{k}^{(S)}$ (see the Supplemental Material [12]), a quantity which remains finite in the classical limit. The scaled gap $\Delta E_{\text {scaled, }}$, plotted vs $H_{z}$ in Fig. 3, is always nonzero for finite values of $S$. However, as $S$ increases, it tends to zero when $\left|H_{z} / H_{\mathrm{SW}}\right|<1$. At an infinite $S$, the reversal is prohibited and the metastable state remains, as

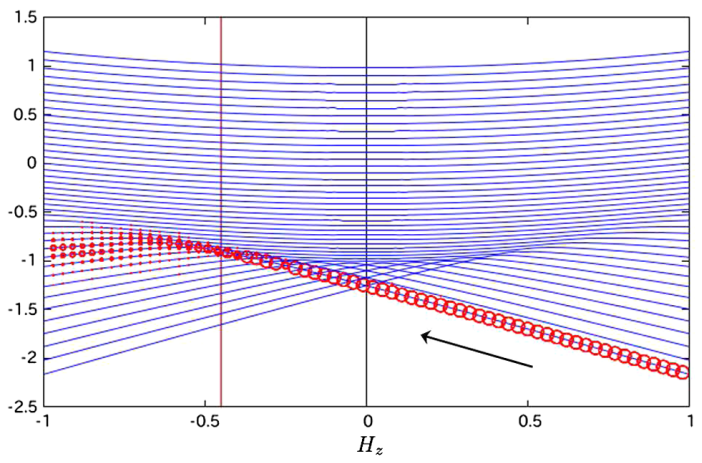

FIG. 2. Energy spectrum for $S=20$ with $D=1$ and $H_{x}=1$ as a function of $H_{z}$. The field is swept from right to left $\left(H_{z}=1-0.004 t\right)$. For each value of $H_{z}$ there are $2 S+1=$ 41 eigenvalues which are plotted by blue lines. The brown vertical line indicates the SW field at which the metastable to stable transition takes place. The red circle denotes populations $\left|c_{n}(t)\right|^{2}$ with $\Psi(t)=\sum_{n} c_{n}(t) \psi_{n}(t)$, where $\psi_{n}(t)$ are eigenstates of the time-dependent Hamiltonian. The radius of each circle denotes the population of the state. 
expected in the classical limit. For $\left|H_{z} / H_{\mathrm{SW}}\right|>1, \Delta E_{\text {scaled }}$ becomes nonzero with a maximum slope near the classical switching field value $H_{\mathrm{SW}}$. Such behavior, related to the transition of the classical SW model (Fig. 1), results from criticality of the scaled gaps.

The $S$ dependence of the field variations of the scaled gaps will now be rapidly investigated. In the mean-field approach of a spinodal phase transition, the probability of a classical system of size $N$ near the spinodal point $H=H_{\mathrm{sp}}$ follows the scaling plot $p(N)=N^{-1 / 3} g\left(\left(H-H_{\text {sp }}\right) N^{2 / 3}\right)$ [13]. Our present spin $S$ could be regarded as the size $N$ of a spinodal system in which single-spin reversals during the spin-diffusion process correspond to the spin transitions at the different crossings of the metastable branch (spin $S$ ) with the intercepted branches (spin $-S+k$ ). This suggests that the data of Fig. 3 should be plotted according to the scaling form: $p^{(S)}=S^{-1 / 3} g\left(\left(H-H_{\mathrm{SP}}\right) S^{2 / 3}\right)$, where $p^{(S)}=1-\prod \exp \left[-\pi\left(\Delta E_{k}^{(S)}\right)^{2} / 2 \hbar c^{(S)} \Delta m_{k}^{(S)}\right] \quad$ (see the Supplemental Material [12]). For a large spin, the gaps are vanishingly small and the exponential can be expanded, leading to $(S \Delta E)^{2} \propto S^{-1 / 3} g\left(\left(H-H_{\mathrm{SP}}\right) S^{2 / 3}\right)$. This is precisely the scaling form for which the numerical data collapse (see the inset of Fig. 3), showing that the quantum SW transition obeys a spinodal phase transition.

We now switch to the last part of this Letter, which relates to magnetization beatings. When the applied field is swept from right to left in Fig. 2, after the SW points, beatings of magnetizations are observed (see Fig. 4). The fast oscillations are simple precessions (as in Fig. 1), whereas the beatings are modulations of these precessions. The amplitude of $s_{z}(t)$ decreases as the field becomes large because a large $H_{z}$ induces a tilt of the precession plane towards the $x y$ plane. The $x y$ components keep the same amplitude representing the rotation around the $z$ axis. Here, we find that all three of the magnetization components show the same beating period, which means that the spin length $s_{f}(t) \equiv\left\langle s_{x}(t)\right\rangle^{2}+\left\langle s_{y}(t)\right\rangle^{2}+\left\langle s_{z}(t)\right\rangle^{2}$, which

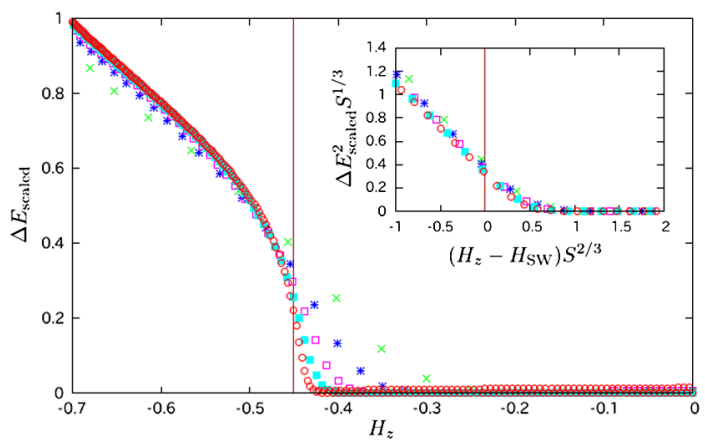

FIG. 3. Normalized energy gaps at avoided-level crossings plotted vs the sweeping magnetic field for $S=20$ (green), 40 (blue), 80 (purple), 160 (cyan), and 320 (red) with $H_{x}=1$, $D=1$. (Inset) Scaling plot showing a mapping of the LZ transition to a dynamical spinodal phase transition. we call spin-length fidelity, also beats in contrast to the spin modulus $S(S+1)$, which is, of course, conserved. This beating is a new characteristic of large, but not infinite, spin systems. As will now be shown, it originates from a new type of quantum phase factor.

The time evolution of the state is given by the timedependent Schrödinger equation $i d \Psi / d t=\mathcal{H} \Psi$, where $\mathcal{H}$ is the scaled Hamiltonian (2), with $H_{z}^{(0)}=0$ for simplicity. Introducing the unitary transformation $U$,

$$
\Psi=U \phi, \quad \text { with } \quad U=\exp \left[i\left(D s_{z}^{2} t-\frac{1}{2} c s_{z} t^{2}\right)\right],
$$

the time evolution is given by

$$
i \frac{\partial}{\partial t} \phi=-H_{x} U^{\dagger} s_{x} U \phi \equiv \mathcal{H}^{\prime} \phi
$$

where

$$
\mathcal{H}^{\prime}=-H_{x}\left\{s_{x} \cos \Theta(\tau)-s_{y} \sin \Theta(\tau)\right\} e^{-i D \tau / S},
$$

with

$$
\Theta(\tau) \equiv \frac{1}{2} v \tau^{2}-2 D s_{z} \tau
$$

(see the Supplemental Material [12]). The Hamiltonian (8) consists of the Zeeman term of a rotating field with the classical-like phase given by expression (9) (see the Supplemental Material [12]), multiplied by a quantum phase term $e^{-i D \tau / S}$ (or $e^{-i D t / S^{2}}$ in the $t$ representation). The first term, simply the function of the scaled spins $s_{x}, s_{y}, s_{z}$, is independent of $S$ and thus tends to the classical model in the $S \rightarrow \infty$ limit. It represents the classical fast precession motion of Fig. 1. The second term, $e^{-i D \tau / S}$, depends on $S$ and tends to unity in the $S \rightarrow \infty$ limit. It is of a genuine quantum origin and is responsible for the observed beatings. Its time $\left(T_{\tau}\right.$ or $\left.T_{t}\right)$ or field $\left(T_{H_{z}}\right)$ period are given by

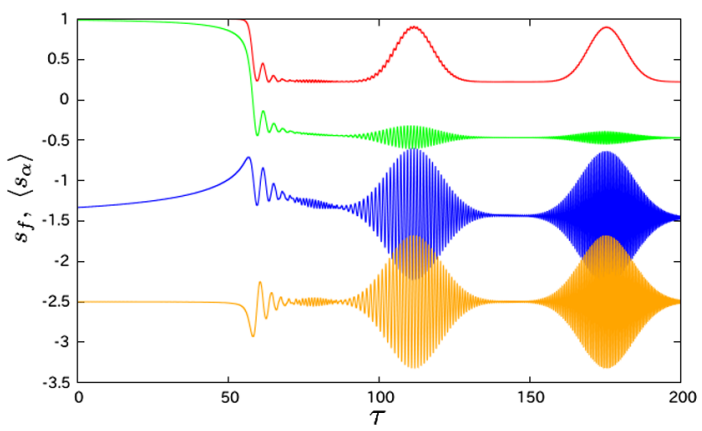

FIG. 4. Beating observed after the SW point. The red line shows the fidelity $s_{f}$ and the green, blue, and orange lines show $\left\langle s_{z}\right\rangle,\left\langle s_{x}\right\rangle-1.5$, and $\left\langle s_{y}\right\rangle-2.5$, where the parameters are $S=20$, $H_{x}=1, D=1$, and $v=0.08$. 

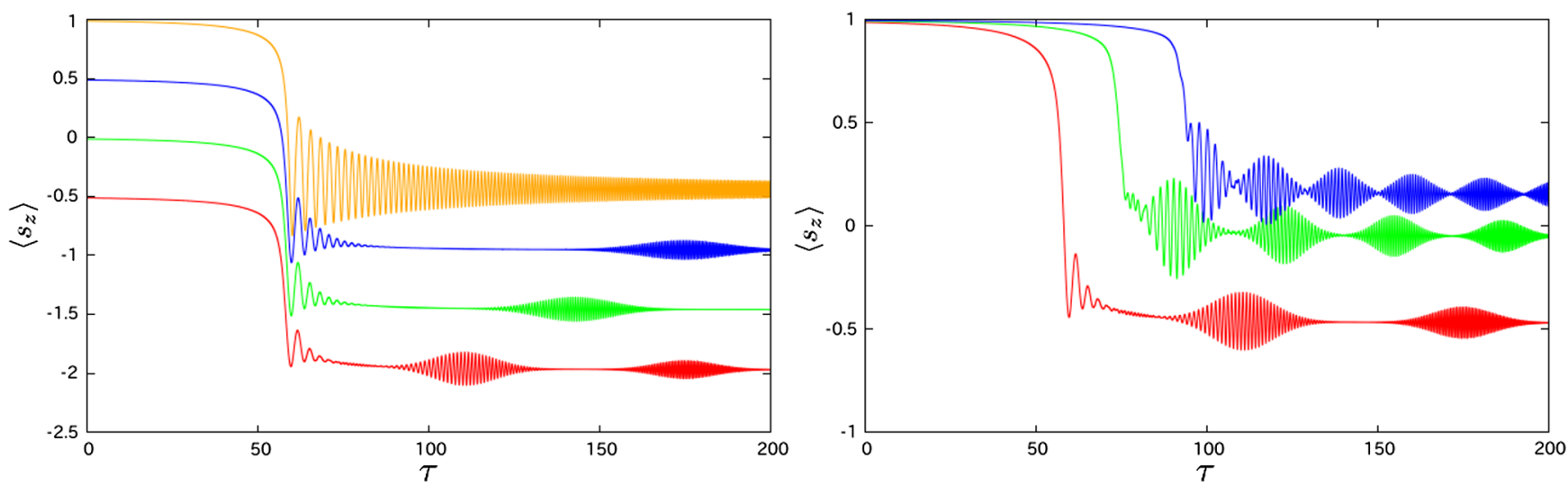

FIG. 5. Dependence of $S_{z}(t)$ for different values of (left panel) spin $S=20$ (red curve), 30 (green curve), 40 (blue curve), and $\infty$ (orange curve) for $v=0.08$ and $D=1.0$, and (right panel) anisotropy constant $D=1$ (red curve), 2 (green curve), and 3 (blue curve) for $S=20$ and $v=0.08$.

$$
T_{\tau}=\frac{2 \pi S}{D} \equiv \frac{T_{t}}{S} \equiv \frac{T_{H_{z}}}{v}
$$

This dependence is confirmed in the simulations (see Fig. 5), showing unambiguously that the beatings are due to the factor $e^{-i D \tau / S}$. Physically, this period is understood as follows. At a given $H_{z}$, ignoring the transverse field, the spin components oscillate at the frequencies $\Delta E(k)=H / S+D / S^{2}+2 D k / S^{2}$ given by the energy difference between neighboring levels with $S_{z}=k$ and $(k+1)$. The difference $|\Delta E(k+1)-\Delta E(k)|=2 D / S^{2}$ gives the above mentioned period.

Finally, we should note that even if the sweeping of the field is stopped and kept constant (see Fig. 6, left panel), the beating persists in the same way. If the field sweeps back, the beating disappears when the field goes beyond the SW point (see Fig. 6, right panel).

In conclusion, we have studied the dynamics of the classical and quantum SW models, showing surmountable and impassable bridges between the two and their identification when $S \rightarrow \infty$. The two most important results consist of the following. (i) The description of the quantum SW model in terms of a dynamical spinodal phase transition with a scaling of the tunneling gaps (or, equivalently, of the time associated with the tunneling proba-

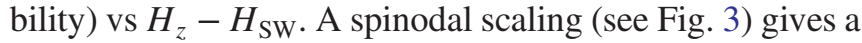
synthetic representation of this complex physics, paving the way for more general studies on the quantum to classical transition. (ii) When the sweeping field is larger than the critical SW field, the spin motion, classically described by a time-dependent precession about its slowly moving local field, becomes modulated in time, leading to a beating of the three spin components and of the spin-length fidelity, with the characteristic period $2 \pi S / D$ obtained analytically thanks to a unitary transformation, allowing one to decompose the classical motion and the quantum mechanical phase terms. These new findings should be instructive in finding new directions of research on general aspects of quantum nanomagnetism beyond molecular magnets; see, e.g., Ref. [14].
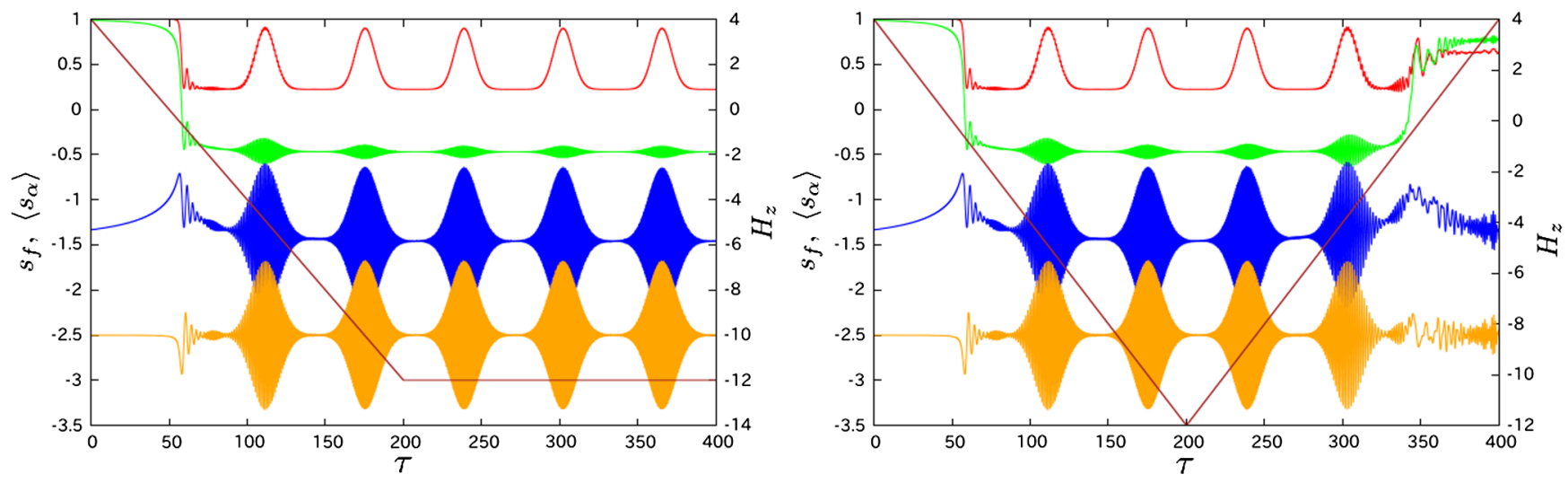

FIG. 6. Beating of the spin-length fidelity, and of the three spin components in a sweeping field above the SW transition for $S=20$ for $D=1, H_{x}=1$ with the sweeping velocity $v=0.08$ until $H_{z}(t)=-12$. (Left panel) Beating is not altered after the field (the brown curve) is set constant. (Right panel) Beating is suppressed after the field (the brown curve) is increased again and reenters above $\left|H_{\mathrm{SW}}\right|$. 
The present work was supported by Grants-in-Aid for Scientific Research C (Grant No. 25400391) from MEXT of Japan, and the Elements Strategy Initiative Center for Magnetic Materials under the outsourcing project of MEXT. The numerical calculations were supported by the supercomputer center of ISSP of the University of Tokyo. T. H. is supported by the Program for Leading Graduate Schools: Material Education program for the future leaders in Research, Industry, and Technology (MERIT) from JSPS, and the JSPS Core-to-Core Program: Nonequilibrium dynamics of soft matter and information.

*Corresponding author. hatomura@spin.phys.s.u-tokyo.ac.jp

[1] E. C. Stoner and E. P. Wohlfarth, Phil. Trans. R. Soc. A 240, 599 (1948).

[2] J. L. Van Hemmen and A. Sütö, in Quantum Tunneling of Magnetization-QTM '94, edited by L. Gunter and B. Barbara, NATO Advanced Study Institutes, Ser. E, Vol. 301 (Kluwer, Dordrecht, 1995), p. 19.

[3] S. A. Owerre and M. B. Paranjape, Phys. Rep. 546, 1 (2015).

[4] D. Gatteschi, R. Sessoli, and J. Villain, Molecular Nanomagnets (Oxford University Press, New York, 2011).

[5] L. Thomas, F. Lionti, R. Ballou, D. Gatteschi, R. Sessoli, and B. Barbara, Nature (London) 383, 145 (1996); B. Barbara, Phil. Trans. R. Soc. A 370, 4487 (2012).
[6] L. D. Landau, Phys. Z. Sowjetunion 2, 46 (1932); C. Zener, Proc. R. Soc. London Sect. A 137, 696 (1932); E. C. G. Stükelberg, Helv. Phys. Acta 5, 369 (1932); E. Majorana, Nuovo Cimento 9, 43 (1932).

[7] S. Miyashita, J. Phys. Soc. Jpn. 64, 3207 (1995); 65, 2734 (1996); H. De Raedt, S. Miyashita, K. Saito, D. Garcia-Pablos, and N. Garcia, Phys. Rev. B 56, 11761 (1997).

[8] W. Wernsdorfer and R. Sessoli, Science 284, 133 (1999); M. Ueda, S. Maegawa, and S. Kitagawa, Phys. Rev. B 66, 073309 (2002).

[9] S. Miyashita, H. De Raedt, and B. Barbara, Phys. Rev. B 79, 104422 (2009).

[10] For the Hamiltonian, we took form (2), which is convenient for studying the classical limit. The present $D$ and $\boldsymbol{H}$ are regarded as $D S^{2}$ and $\boldsymbol{S H}$, respectively, for the usual quantum Hamiltonian $\mathcal{H}=-D S_{z}^{2}-\boldsymbol{H} \cdot \boldsymbol{S}$.

[11] Y. Kayanuma and S. Fukuchi, J. Phys. B 18, 4089 (1985); S. Brundobler and V. Elser, J. Phys. A 26, 1211 (1993); A. V. Shytov, Phys. Rev. A 70, 052708 (2004).

[12] See Supplemental Material at http://link.aps.org/ supplemental/10.1103/PhysRevLett.116.037203 for the scaling form of energy gaps and the derivation of Eq. (8).

[13] T. Mori, S. Miyashita, and P. A. Rikvold, Phys. Rev. E 81, 011135 (2010).

[14] I. G. Rau et al., Science 344, 988 (2014); A. Namai et al., Nat. Commun. 3, 1035 (2012). 